\title{
Human umbilical cord mesenchymal stem cells (hUCMSCs) promotes the regeneration of severe endometrial damage in a rat model
}

\section{Zhuang Mei}

Shanghai University of Traditional Chinese Medicine

\section{Zhang Wuwen}

Shanghai University of Traditional Chinese Medicine

Liu Dan

Shanghai University of Traditional Chinese Medicine

Yan Hua

Shanghai University of Traditional Chinese Medicine

\section{Fang Ge}

Shanghai University of Traditional Chinese Medicine

\section{Heng Boon Chin}

Peking University

\section{Sun Yan}

\section{Fudan University}

Tong guoqing ( $\square$ drivftongguoqing@medmail.com.cn )

Reproductive medicine center https://orcid.org/0000-0001-9041-5530

\section{Research article}

Keywords: human umbilical cord mesenchymal stem cells, severe endometrial damage, infertility, animal models

Posted Date: January 6th, 2020

DOI: https://doi.org/10.21203/rs.2.20062/v1

License: (c) (i) This work is licensed under a Creative Commons Attribution 4.0 International License. Read Full License 


\section{Abstract}

Background: Intrauterine adhesions (IUA) is a common endometrial disease, which is one of the causes of infertility. Transplantation of stem cells may provide a viable solution for endometrial repair and regeneration. We made a model of severe endometrial injury in rats, transplanted hUCMSCs, and studied the effect of hUCMSCs on endometrial regeneration. Methods: Thirty-two female Sprague-Dawley rats were randomly divided into four groups: normal group, injury control group, MSC1 group and MSC2 group. After 15 days of intervention and transplantation, histological analysis was performed and cytokine messenger RNA expression was measured. Results: The HE staining results showed that the endometrial tissue of the injury control group was significantly damaged, and the endometrial tissues of the MSC1 group and the MSC2 group were improved. We did not detect the expression of keratin and vimentin in the injury control group. However, there was the expression of keratin and vimentin in the MSC1 group and the MSC2 group. The results of Real-time PCR showed that the expression levels of tumor necrosis factor-a (TNF-a) mRNA in the normal group and MSC1 group was lower than that of the injury control group $(P<0.05)$. The expression levels of basic fibroblast growth factor (bFGF) mRNA in the normal group and MSC2 group were higher than that of the injury control group $(P<0.05)$. The expression levels of interleukin-1 $\beta$ (IL-1 $\beta$ ) mRNA in the normal group was lower than that of the control group $(P<0.05)$. Conclusions: Transplantation of hUCMSCs promoted the recovery of severe endometrial damage in rats. These findings suggest the effect may be related to the mechanisms of homing and paracrine secretion.

\section{Background}

Due to the influence of various social and environmental factors, the incidence of infertility is increasing, and the application of assisted reproductive technology (ART) is becoming more and more widespread. Although the current clinical pregnancy rate of in vitro fertilization (IVF) is $40-60 \%{ }^{[1,2]}$, even in some successful centers, some couples still face the difficulty of repeated implant failure.Previous studies have shown that one-third of embryonic failures are due to a reduction in endometrial receptivity [3]. Endometrial receptivity refers to the capacity of the endometrium to accept embryos. Intrauterine adhesions, also known as Asherman syndrome (AS), is one of the most common causes of decreased endometrial receptivity after intrauterine surgery or inflammation. At present, most experts believe that IUA should be defined as intrauterine adhesion or fibrosis, which is accompanied by one or more of the following clinical symptoms: menstrual reduction, amenorrhea, repeated abortion, infertility and abnormal placental formation. If there is no such symptom, it is called asymptomatic intrauterine adhesion ${ }^{[4-5]}$. There are three processes in the routine treatment of IUA: firstly, surgical adhesion is used to separate adhesions and enable recovery of uterine cavity morphology. Secondly, intrauterine devices and balloon stents, are placed in the uterus to prevent adhesion recurrence. Finally, normal menstruation needs to be restored. IUA may also be caused by fibrosis, and poor capacity for uterine intima repair and regeneration. 
The main medical treatment modalities for endometrial repair are as follows: (1) Estrogen administration: Estrogen can increase the residual endometrium in the uterine cavity, while preventing the formation of new lesions, thereby improving the uterine environment. (2) Administration of aspirin or sildenafil: Weckstein ${ }^{[6]}$ suggested the use of low doses of aspirin. Sher ${ }^{[7]}$ suggested that the use of sildenafil can increase endometrial blood flow and intima thickness. (3) Integrated Chinese and Western medicine treatment: Some Chinese medicine doctors believe that combined with traditional Chinese medicine, acupuncture and other treatments can increase endometrial blood flow and improve endometrial receptivity. (4) Stem cell therapy.

Currently utilized stem cell types in animal experiments and clinical trials include bone marrow mesenchymal stem cells (BMSCs) ${ }^{[8-10]}$, adipose-derived mesenchymal stem cells (ADSCs) ${ }^{[11]}$, and menstrual blood-derived stromal cells (menSCs) ${ }^{[12]}$, and human amniotic mesenchymal stromal cells (hAMSCs) ${ }^{[13]}$. The several aforementioned stem cells have been confirmed to have some efficacy on endometrial regeneration in related experiments.

However, these aforementioned stem cells have various deficiencies such as invasive isolation and low purity. Utilizing hUCMSCs may overcome these limitations, and these cells do not face the problem of decreased differentiation potential with increasing patient age ${ }^{[14]}$. Additionally, hUCMSCs are easily isolated from a readily available medical waste product, have low risks of viral infection, and have high proliferative potential within in vitro culture. These aspects are superior to invasively extracted bone marrow mesenchymal stem cells. Mesenchymal stem cells(MSCs) derived from the umbilical cord can be obtained from the amniotic membrane, cord lining, Wharton's jelly, and perivascular region ${ }^{[15]}$. HUCMSCs have lower immunogenicity than bone marrow mesenchymal stem cells ${ }^{[16]}$. Due to the biological characteristics and immunological characteristics of hUCMSCs, an increasing number of research studies have applied these cells to the treatment of various systemic diseases.

Our goals are to explore the pathological changes of endometrium in rats, elucidate the regeneration effect of transplanted hUCMSC on severely damaged endometrium, study its possible mechanism of action through changes in cytokine gene expression, and explore the treatment of severe people An effective and feasible method for endometrial injury.

\section{Methods}

Animals

In this experiment, 32 SD rats, SPF grade, 8-9 weeks old, weighing 160-220 g, were provided by the Animal Experimental Center of Shanghai University of Traditional Chinese Medicine. Animal were maintained in cages (4/cage), at an ambient temperature of $26^{\circ} \mathrm{C}$, humidity of $50 \%-60 \%, 12$ hours of light and darkness daily, and given free access to food and drinking water. This experimental study was carried out in accordance to the "Guidelines for the Protection and Application of Experimental Animals" issued by the National Institute of Health in the United States. The use of laboratory animals strictly 
follow the corresponding regulations of the Animal Experimental Management Committee of the Shanghai University of Traditional Chinese Medicine.

\section{Culture of hUCMSCs}

The hUCMSCs used in this experiment were obtained as a gift from from the Shandong Stem Cell Group. After thawing the hUCMSCs, the cells were cultured in Dulbecco's modified Eagle's medium (DMEM, GIBCO) medium containing $10 \%$ fetal bovine serum( $\mathrm{FBS}, \mathrm{GIBCO}$ ), in an incubator at $37^{\circ} \mathrm{C}, 5 \% \mathrm{CO}_{2}$ and $40 \%-60 \%$ saturated humidity. Thereafter, the culture medium was refreshed every $2-3$ days, until cells formed a confluent monolayer. Serial passage of hUCMSCs was carried out with $0.25 \%(\mathrm{w} / \mathrm{v})$ trypsin $(0.02 \%$ EDTA).

\section{Flow Cytometry}

hUMSCs were phenotypically characterized by flow Cytometry (BECKMAN COULTER). Briefly, the cells in each tube $\left(1.0 \times 10^{5}\right)$ were labelled with the following monoclonal antibodies: CD146, CD140, CD105, CD29 and CD44. The results of flow cytometry were analysed by the FlowJo® software.

\section{Rat Model of Severe Endometrial Damage}

The model of severe endometrial injury in rats was made with reference to Jing ${ }^{[8]}$. Intrauterine perfusion of absolute ethanol caused severe damage to the endometrium. Endometrial injury surgery is performed under anesthesia. The operation of abdominal anesthesia is simple and safe. The anesthesia time can be maintained for one hour. The choice of narcotic drugs is sodium pentobarbital, the dosage is small, and the mortality from anaesthesia is low. On the morning of surgery day,rats were anesthetized with $3 \%$ $(\mathrm{w} / \mathrm{v})$ pentobarbital sodium at a dose of $40 \mathrm{mg} / \mathrm{kg}$. The lower abdomen was cut longitudinally and the skin was layered into the abdominal cavity. The rat uterus is $\mathrm{Y}$-shaped, and the arterial clip is clamped at the right uterine horn and the right uterine cavity (the arterial clip is toothless, no damage to the uterus). The needle $(1 \mathrm{ml})$ is extended into the uterine cavity and $95 \%(\mathrm{v} / \mathrm{v})$ absolute ethanol was injected into the uterine cavity and maintained the filling state of the uterine cavity for $6 \mathrm{~min}$. After $6 \mathrm{~min}$, the ethanol in the uterine cavity was aspirated. The left uterus is treated in the same way. After flushing the abdominal cavity with $0.9 \%$ sodium chloride solution, the abdominal cavity and skin were sutured layer by layer.The entire operation takes about 20-25 minutes.After the operation, the rats were placed in an incubator to maintain their body temperature. After waking up, they were returned to their cages and fed with highenergy feed for 1 day and kept free to take water. On the day of surgery, 80,000 units of penicillin were injected intramuscularly into each rat.

\section{Transplantation of hUCMSCs}

Thirty-two SD rats were randomly divided into 4 groups of 8 rats each: normal group(NG), injury control group (ICG), MSC1 group (MG1) and MSC2 group (MG2) (Fig. 1). The normal group did not undergo any treatment. In the ICG, a severe model of rat endometrial damage was established by the above method. In 
the MG1, under isoflurane inhalation anesthesia,the hUCMSCs were injected into the tail vein within 24 hours after the model was established ( $1 \times 10^{7}$ cells ). In the MG2 within 24 hours after the model was established each rat was treated in the same way as the MG1.Then, on the 7th day after the model was established, rats in MG2 were again subjected to laparotomy under abdominal anesthesia. During the operation, hUCMSCs were injected into the uterine cavity with a $1 \mathrm{ml}$ syringe $\left(1 \times 10^{7}\right.$ cells per side of the uterine cavity). On the 15th day after modeling, 32 rats were sacrificed by cervical dislocation.

Hematoxylin-Eosin Staining

Rat uterus were fixed in $4 \%(\mathrm{w} / \mathrm{v})$ formaldehyde, and these uterus were cut into tissue sections with a thickness of about $4 \mathrm{~mm}$. The cut surface was a cross section and were placed vertically in an embedding cassette. The water in the tissue block was removed with an alcohol gradient at a concentration of $50 \% \rightarrow 70 \% \rightarrow 80 \% \rightarrow 90 \% \rightarrow 95 \%$. Then, the tissue block was placed in the clearing agent xylene I, II for $2 \mathrm{~h}$, followed by dissolved paraffin, and then in an embedded wax block, prior to being cut into thin slices (thickness, $4 \mu \mathrm{m}$ ), and subjected to the process of Hematoxylin-Eosin Staining.

Immunohistochemistry

The sections were immersed in xylene to remove paraffin from the sections, and then rehydrated by immersion in high concentration to low concentration alcohol. For antigen retrieval, the sample was immersed in citrate buffer $(0.01 \mathrm{M}, \mathrm{pH} 6.0)$, boiled for $10 \mathrm{~min}$, and naturally cooled. After rinsing 3 times with PBS for 2 minutes each time, the samples were incubated with primary antibody at room temperature for 60 minutes, and rinsed 3 times with PBS for 2 minutes each time. Then the samples were incubated with detection reagent (Beijing Jinqiao Biological) at room temperature for 30 minutes, rinse again with PBS for 3 times, and color was developed vy exposure to horseradish peroxidase (DAB, Shanghai Shenggong Biological Company) for 5-10 minutes, followed by counterstaining with hematoxylin, dehydration, and sealing with neutral resin.

Real-time PCR (q-PCR)

RNA was extracted from excised uterines using the RNAiso Plus kit (BID-RAD) according to the manufacturer's instructions. As previously described, CDNA was prepared and amplified by SYBR Greenbased relative quantification PCR using the ABI StepOne machine. We processed one excised uterine sample at a time, utilizing GAPDH as an internal reference. Results of the q-PCR showed that the relative variation of gene expression between groups, which was analyzed by the $2^{-\triangle \Delta C T}$ method. Data was computed from three different experiments. The primer sequences used in this study are as follows:

Primers are designed using Primer 5.0 software(Shanghai Biosynthesis).

TNF-a:

Forward primer 5'-CCTCTTCTCATTCCTGCTCGT-3' 
Reverse Primer 5'-GGCCATGGAACTGATGAGAGG-3'

bFGF:

Forward primer 5'-CCCACACGTCAAACTACAGCTC-3'

Reverse Primer 5'-TAACACACTTAGAAGCCAGCAG-3'

IL-1ß:

Forward primer 5'-AATGCCTCGTGCTGTCTGACC-3'

Reverse Primer 5'-GCCACAGGGATTTTGTCGTT-3'

IL-6:

Forward primer 5'-CAGCCAGTTGCCTTCTTGGGA-3'

Reverse Primer 5'-TGCACAACTCTTTTCTCATTTCCA-3'

GAPDH:

Forward primer 5'-ACCACAGTCCATGCCATCAC-3'

Reverse Primer 5'-TCCACCACCCTGTTGCTGTA-3'

Data Analysis

The uterus of all 32 rats were subjected to Hematoxylin-Eosin staining and endometrial cell marker protein expression analysis.Seven samples from NG(7/8), eight samples from ICG (8/8), seven samples from MG1 (7/8), and eight samples from MG2 (8/8). Two samples were abandoned due to specimen preservation issues.A total of 30 samples were analyzed by q-PCR. All data was statistically processed using the SPSS 21.0 software package. One-way ANOVA was applied by comparing multiple sets of samples. $\mathrm{P}<0.05$ was considered statistically significant.

\section{Results}

Culture passage of hUCMSCs

After resuscitation, hUCMSCs were oval; after the cells were cultured for 48 hours, the cells began to grow adherently; after 5 days of cell culture, the cells adhered to the wall and were basically covered with medium (Fig. 2).

After cell thawing, hUCMSCs were round in shape (A); after 48 hours of culture in the medium, the cells adhered and began to grow (B); after 5 days of cell culture, the cells formed an almost confluent layer (C). 
Results of flow cytometry showed that the expression levels levels of CD140, CD105, CD29 and CD44 were $96 \%, 93.7 \%, 97.3 \%$ and $97 \%$ respectively (Fig. 3). The expression levels of these marker genes were the same as those reported by previous MSC-related research. Therefore, our cultured hUCMSCs are considered to have high purity. Within in vitro culture, hUCMSCs maintained the characteristic morphological phenotype of MSCs (Fig. 2).

Flow cytometry showed that the expression levels of CD140 was 96\%, the expression levels of CD105 was $93.7 \%$, the expression levels of CD29 was $97.3 \%$, and the expression levels of CD44 was $97 \%$ in hUCMSCs.

\section{Rat Endometrial Hematoxylin-Eosin Staining}

In the normal group (NG), the endometrial epithelium was cubic columnar in morpology, and the intima was wrinkled. The endometrial glands and interstitium can be observed under the epithelium. In the injury control group (ICG), the uterine cavity was closed. There was no endometrial epithelium, glandular and endometrial stroma, but fibrovascular proliferation, and some scattered inflammatory cells were observed. In the MSC1 Group (MG1), epithelial cells can be observed in the uterine cavity, and the folds were reduced. The endometrial stromal cells, individual glands and scattered inflammatory cells can be observed. In the MSC2 group (MG2), the endometrial epithelium was cubic columnar in morphology, the intima was wrinkled, and the endometrial glands and interstitiaum can be observed under the epithelium. A small number of scattered inflammatory cells were seen in the interstitium(Figure 4).

NG: The endometrial epithelium was cubic columnar in morphology and the intima was wrinkled. ICG: Endometrial injury, closed uterine cavity, no endometrial epithelium, glands and endometrial stroma. MG1: epithelial cells were seen in the uterine cavity, wrinkles decreased, endometrial cells and individual glands observed, regeneration of endometrial damage observed. MG2: Endometrial morphology and he normal group was similar and the endometrial damage was repaired significantly.

Rat endometrial cell marker protein expression

Keratin (CK) is expressed in epithelial cells to maintain the integrity and continuity of epithelial tissue. CK was confirmed to be expressed in the epithelial cells, as shown by immunohistochemical staining of the endometrium. Vimentin is expressed in various cell types such as mesenchymal cells, vascular endothelial cells, and smooth muscle cells. In this study, CK immunohistochemical staining was used to observe endometrial and glandular epithelial cells; while Vimentin immunohistochemical staining was used to observe endometrial stromal cells.

The results of CK immunohistochemical staining were as follows: In the normal group, there were a large number of brown-yellow granules in the endometrial epithelium and glandular epithelium, and CK was positively expressed (Fig. 5NG-A); There were no endometrial epithelium and glands in the injury group, and CK was negatively expressed (Fig. 5ICG-A); In the MG1 group, a small amount of brown particles were observed within the uterus in the endometrial epithelium, and CK was weakly expressed (Fig. 5MG1- 
A). In the MG2 group, a small amount of brown-yellow particles were observed in the columnar epithelium of the endometrium, and CK was weakly expressed (Fig. 5MG2-A).

The results of Vimentin immunohistochemical staining were as follows: In the NG group (Fig. 5NG-B), the dark brown particles within the endometrial stroma of the endometrial epithelium were densely distributed. In the ICG group (Fig. 5ICG-B), the uterine cavity was closed, and the scattered brown particles were distributed in the endometrial stroma, with interstitial edema being observed. In the MG1 group (Fig. 5MG1-B), the brown particles in the endometrial stroma were evenly distributed under the endometrial epithelium. The distribution density of brown particles was thinner than that in the NG group (Fig. 5NG-B). In the MG2 group (Fig. 5MG2-B), the endometrial epithelium and glandular epithelium were deep within the endometrial stroma. The brown particles are similarly distributed and the image is similar to the NG group (Fig. 5NG-B).

The expression levels of bFGF, TNF- $a$, IL- 6 and IL-1 $\beta$ genes in the rat endometrium

The expression levels of bFGF mRNA was lowest in the ICG, which was different from the NG $(P<0.05)$. The expression levels of bFGF mRNA in the MG1 was higher than that of the ICG, but there was no statistically significant difference between the MG1 and ICG $(P>0.05)$. There were no significant differences between the MG1, MG2 and NG(P>0.05). The expression levels of TNF-amRNA was highest in the ICG, which was different from the NG $(P<0.05)$. The expression levels of the MG1 was lower than that of the ICG $(P<0.05)$, and the expression levels of the MG2 was lower than that of the ICG $(P<0.01)$. A significant difference was not found between the three groups of NG, MG1 and MG2 ( $P>0.05)$. The expression levels of IL-6 mRNA in the NG was the highest, and there were no statistically significant differences between the four groups $(P>0.05)$. The expression levels of IL-1 $1 \beta$ mRNA in the ICG was the highest, its expression levels was significantly different from NG $(P<0.05)$. The expression levels of the MG1 and MG2 were lower than that of the ICG, but there was no statistically significant difference between these groups $(P>0.05)$ (Figure 6).

The results of bFGF mRNA expression: the expression levels of the normal group and the MSC2 group was higher than that of the control group $(P<0.05)$. The result of TNF-a mRNA expression: Expression levels of the normal group and MSC1 group was significantly lower than that of the control group $(\mathrm{P}<$ 0.05); while the expression levels of the MSC2 group was significantly lower than that of the control group as well $(P<0.01)$. The results of IL-6mRNA expression: No significant differences were found amongst the four groups $(P>0.05)$. The results of IL-1 $\beta$ mRNA expression: Expression levels of the normal group was lower than that of the control group $(P<0.05)$.

\section{Discussion}

Stem cell therapy is becoming a new and effective therapeutic modality for endometrial repair and regeneration, with extensive research and clinical trials. MSCs are a subpopulation of adult stem cell that have multi-lineage differentiation potential, low immunogenicity and certain immune-regulatory functions 
[19]. MSCs express marker genes of CD105, CD73, CD44, CD166, CD54, CD102, CD49, but do not express the marker gene of CD14, CD34, CD 45, CD11a and HLA-DR, and lack expression of the defining markers of red blood cells, platelets and endothelial cells such as CD31 ${ }^{[20-21]}$. Due to the low expression of major histocompatibility complex (MHC) I molecules and lack of MHC II molecule expression by MSCs, cytotoxic T lymphocytes and natural killer cells are unable to recognize and destroy allogeneic MSCs [2223]. In this study, hUCMSCs highly express the marker genes of CD140, CD29, CD44 and CD105, which are consistent with MSCs characterization markers. The low immunogenicity of hUCMSCs is the major advantage of its utilization in allogeneic transplantation. This makes it possible to achieve allogeneic transplantation without using immunosuppressive drugs, which is one of the theoretical basis of this study.

The severe endometrial damage model was established as described by a previous study ${ }^{[10]}$. The principle mode of action is cellular dehydration and protein coagulation. The arterial clip was placed at the uterine horn and uterus bifurcation to prevent the overflow of absolute ethanol. After 6 minutes of treatment time, the ethanol in the uterine cavity was aspirated, but the uterine cavity was not washed, so that severe damage to the endometrium can be inflicted.

HUCMSCs are derived from medical waste from a wide range of sources, are conveniently and noninvasively isolated, and there is no ethical controversy over its procurement, so it is a suitable source for stem cell therapy. Ding ${ }^{[24]}$ transplanted umbilical cord mesenchymal stem cells cultured in vitro into the infracted brains of rats. The results showed that umbilical cord mesenchymal stem cells exerted a positive neuroregenerative effect on the cerebral infarction area. Ryu ${ }^{[25]}$ found that there were no significant differences in the therepautic efficacy of MSCs from different sources in the treatment of spinal cord injury in dogs. However, hUCMSCs are more potent in inducing nerve regeneration than MSCs from other sources and also exhibit higher anti-inflammatory activity.

In this experiment, the main experimental indicators were Hematoxylin-Eosin staining of rat endometrium and marker protein expression of endometrial cells, and to understand the repair effect of hUCMSCs transplantation on severe endometrial injury. The secondary experimental indicator was q-PCR analysis of endometrial cytokines to understand the possible mechanism of action of stem cells.

After establishing a model of severe endometrial injury, hUCMSCs were administered by tail vein injection, or tail vein injection combined with intrauterine infusion. The rat uterus was harvested 15 days after injury. The HE staining results of the MG1 and MG2 were compared with the HE staining results of the ICG. The presence of the uterine cavity was observed, and the endometrial epithelium and gland were also observed. Compared with MG1, MG2 clearly observed the morphology of the endometrial epithelium, increased the number of wrinkles, and increased the number of glands. The immunohistochemical staining results showed that there was negative expression of CK protein in the control group, weak expression of CK protein in the MG1, and positive expression of CK protein in the MG2. The distribution of vimentin in the injured control group was loose and scattered, while the distribution of vimentin in the MG1 was evener than that of the control group. However, the expression in the MG1 was lower than that 
of the normal group, and the vimentin in the MG2 were densely distributed under the endometrial epithelium, which was similar to the normal group. Therefore, our results demonstrated that hUCMSCs can promote the repair of severe endometrial damage in rats, and the effect of intravenous injection combined with local application in the uterine cavity is better than intravenous injection.

The mechanism of action of MSC in tissue regeneration has not been clarified. It is currently believed that the relevant mechanisms of action include homing differentiation, paracrine function, and immunomodulatory effects. Most researchers believe that stem cell homing is similar to leukocyte migration behavior and is a multi-step, multi-factor coordinated process involving many cytokines, receptors, adhesion factors and extracellular matrix-degrading proteases ${ }^{[26]}$. The stromal cell-derived factor-1 (SDF-1)/CXC chemokine receptor 4 (CXCR-4) axis is an important biological axis that promotes homing of mesenchymal stem cells to injured tissues ${ }^{[27]}$. Inflammation, tissue ischemia, liver drug damage, and damage to the body with chemotherapy drugs, as well as pro-inflammatory factors such as TNF-a or IL-1, may cause an increase in SDF-1 expression. The model of severe endometrial injury induced by intrauterine infusion of absolute ethanol was utilized. The expression levels of IL-1ßand TNFamRNA in ICG was higher than that in NG, which may induce hUCMSCs to home into the inflammation and repair the tissue.

The paracrine effects of MSCs help restore injured tissues through various mechanisms, including the production of antioxidants such as catalase, glutathione peroxidase and manganese superoxide dismutase, which can reduce the presence of destructive anaerobic free radicals in ischemic tissue ${ }^{[28]}$. MSCs are a rich source of growth factors such as bFGF, vascular endotjelial growth factor (VEGF), hepatocyte growth factor and insulin-like growth factor-1, which promote angiogenesis, inhibit apoptosis and stimulate cell proliferation ${ }^{[29]}$. Tog ${ }^{[30]}$ intravenously injected BMSCs into an acute renal failure model induced by ischemia-reperfusion in rat kidney. After $24 \mathrm{~h}$, proinflammatory cytokines IL-1 $\beta$ and TNF-a were significantly decreased. Anti-inflammatory cytokines IL-10 and bFGF were Up-regulated.Above changes suggested that the role of MSCs in improving renal function at the early stages of acute renal failure may be related to paracrine effects. The expression levels of TNF-a in MG1 and MG2 group was decreased compared with the ICG group, and there was no significant difference in the expression levels of IL-1 $\beta$ and IL- 6 between the MG1 and MG2. The expression levels of bFGF in the MG2 was higher than that in the ICG. These results suggested that the paracrine effect of hUCMSCs may also play a significant role in endometrial repair.

\section{Conclusions}

In summary, our study demonstrated that transplantation of hUCMSCs promoted the repair of severe endometrial damage in rats, and that the effect of venous combined intrauterine transplantation was better than that of simple vein grafting. The homing and paracrine mechanisms are potentially involved in the endometrial repair process. The molecular mechanisms underlying this process are still not well understood. Future studies are required to further understand the potential impact on the fertility of rats. 


\section{Abbreviations}

hUCMSCs : human umbilical cord mesenchymal stem cells

IL-6 : interleukin-6

IL-1 $\beta$ : interleukin-1 $\beta$

TNF-a : tumor necrosis factor-a

bFGF : basic fibroblast growth factor

ART : assisted reproductive technology

IVF : in vitro fertilization

RIF : repeated implantation failure

IUA : intrauterine adhesions

AS : Asherman syndrom

BMSCs : bone marrow mesenchymal stem cells

ADSCs : adipose-derived mesenchymal stem cells

menSCs : menstrual blood-derived stromal cells

hAMSCs : human amniotic mesenchymal stromal cells

\section{Declarations}

\section{Fundings}

This work was supported by the grants from the National Natural Science Foundation of China (Nos. 81170571 and 81571442$)$.

\section{Ethics Approval and Consent to Participate}

All the related experiment procedures met the requirements of Shanghai University of Traditional Chinese Medicine Animal Testing Ethics Committee (SZY201705006). All the procedures in this study were approved.

\section{Authors' Contributions}


Mei Zhuang and Guoqing Tong designed the work. Wuwen Zhang, Dan Liu, Hua Yan, Ge Fang, Boon Chin Heng and Yan Sun participated in the experiments and analyzed and processed the data. Mei Zhuang and Wuwen Zhan drafted the manuscript. All authors read and approved the final manuscript.

\section{Competing Interest}

The authors declare that they have no competing interests.

\section{Consent for Publication}

Not applicable.

\section{Availability of Data and Materials}

Please contact the corresponding authors for data requests.

\section{Acknowledgements}

We thank members of the laboratory for help and critical reading of the manuscript, and express our thanks particularly to department of Pathology, Shanghai Traditional Chinese Medicine Interated Hospital, for their technical assistance.

\section{References}

1. Margalioth EJ, Ben-Chetrit A, Gal M, et al. Investigation and treatment of repeated implantation failure following IVF-ET. Hum Reprod 2006;21(12):3036-3043 doi:org/10.1093/humrep/del305

2. Rinehart J.Recurrent implantation failure: definition. J Assist Reprod Genet 2007; 24(7):284287 doi:10.1007/s10815-007-9147-4

3. Achache H, Revel A. Endometrial receptivity markers, the journey to successful embryo implantation . Human Reproduction Update,2006,12(6):731-746 doi:10.1093/humupd/dml004

4. Conforti A, Alviggi C, Mollo A, et al.The management of Asherman syndrome: A review of literature. Reprod Biol Endocrinol 2013;11:118 doi:10.1186/1477-7827-11-118

5. Yu D, Wong YM, Cheong Y,et al.Asherman syndrome-one century later.Fertil Steril 2008;89(4):759779 doi:10.1016/j.fertnstert.2008.02.096

6. Weckstein LN, Jacobson A, Galen D, et al. Low-dose aspirin for oocyte donation recipients with a thin endometrium: prospective, randomized study.Fertil Steril 1997;68(5):927-930 doi:10.1016/s00150282(97)00330-0

7. Sher G, Fisch JD. Effect of vaginal sildenafil on the outcome of in vitro fertilization (IVF) after multiple IVF failuresattributed to poor endometrial development.Fertil Steril 2002;78(5): 10731076 doi:10.1016/s0015-0282(02)03375-7

8. Jing Z, Qiong Z, Yonggang W, et al. Rat bone marrow mesenchymal stem cells improve regeneration of thin endometrium in rat. Fertil Steril 2014;101(2):587-594 doi:10.1016/j.fertnstert.2013.10.053 
9. Nagori CB, Panchal SY, Patel H. Endometrial regeneration using autologous adult stem cells followed by conception by in vitro fertilization in a patient of severe Asherman's syndrome. J Hum Repord Sci 2011;4(1):43-48 doi:10.4103/0974-1208.82360

10 . Singh N, Mohanty S, Seth T, et al. Autologous stem cell transplantation in refractory Asherman's syndrome: A novel cell basedtherapy. J Hum Repord Sci 2014;7(2):9398 doi:10.4103/0974-1208.138864

11. Kilic S, Yuksel B, Pinarli F, et al. Effect of stem cell application on Asherman syndrome, an experimental rat model. J Assist Repord Genet 2014;31(8):975-982 doi:10.1007/s10815-0140268-2

12. Tan J, Li P, Wang Q, et al. Autologous menstrual blood-derived stromal cells transplantation for severe Asherman's syndrome. Hum Repord 2016;31(12):2723-2729 doi:10.1093/humrep/dew235

13. Gan L, Duan $\mathrm{H}, \mathrm{Xu} \mathrm{Q}$, et al. Human amniotic mesenchymal stromal cell transplantation improves endometrial regeneration in rodent models of intrauterine adhesions. International Society for Cellular Therapy 2017;19(5):603-616 doi:10.1016/j.jcyt.2017.02.003

14. Endometrial stem/progenitor cells:The first 10 years. Human Reproduction Update 2016;22(2):137163 doi:10.1093/humupd/dmv051

15. Ding DC, Chang YH, Shyu WC, et al. Human umbilical cord mesenchymal stem cells: a new era for stem cell therapy. Cell Transplant 2015;24(3):339-347 doi:10.3727/096368915X686841

16. Wang $M$, Yang $Y$, Yang $D$, et al. The immunomodulatory activity of human umbilical cord bloodderived mesenchymal stem cellsin vitro. Immunology 2009;126(2):220-232. doi:10.1111/j.13652567.2008.02891.x

17. Castro-Manrreza ME, Montesinos JJ. Immunoregulation by Mesenchymal Stem Cells: Biological Aspects and Clinical Applications. J Immunol Res 2015; 2015: 394917 doi:10.1155/2015/394917

18. Klyushnenkova E, Mosca JD, Zernetkina $V$, et al. T cell responses to allogeneic human mesenchymal stem cells: immunogenicity, tolerance, and suppression. J Biomed Sci 2005;12(1) : 47-

57 doi:10.1007/s11373-004-8183-7

19. Barzilay R, Melamed E, Offen D. Introducing transcription factors to multipotent mesenchymal stem cells:making transdifferentiation possible. Stem Cells 2009;27(10):2509-2515 doi:10.1002/stem.172

20. Pittenger MF, Mackay AM, Beck SC, et al.Multilineage potential of adult human mesenchymal stem cells. Science 1999;284( 5411):143 -147 doi:10.1126/science.284.5411.143

21. Spaggiari GM, Capobianco A, Becchetti $S$, et al. Mesenchymal stem cell-natural killer cellinteractions: evidence that activated NK cellsare capable of killing MSCSs, whereas MSCs can inhibit IL-2-induced NK cell proliferation. Blood 2006;107(4) : 1484-1490 doi:10.1182/blood-2005-07-2775

22. Ding DC, Shyu WC, Chiang MF, et al. Enhancement of neuroplasticity through upregulation of beta1integrin in human umbilical cord-derived stromal cell implanted stroke model. Neurobiol Dis 2007;27:339-353 doi:10.1016/j.nbd.2007.06.010

23. Ryu HH, Kang BJ, Park SS, et a Comparison of mesenchymal stem cells derived from fat, bone marrow, Wharton's jelly, and umbilical cord blood for treating spinal cord injuries in dogs. J Vet Med 
Sci 2012;74(12):1617-1630 doi:10.1292/jvms.12-0065

24. Mannello F. Multipotent mesenchymal stromal cell recruitment, migration, and differentiation: what have matrix metalloproteinases got to do with it? Stem Cells 2006; 24(8): 1904-1907. doi:10.1634/stemcells.2005-0608

25. Lapidot T, Dar A, Kollet O. How do stem cells find their way home? Blood 2005;106(6):19011910 doi:10.1182/blood-2005-04-1417

26. Dernbach E, Urbich C, Brandes RP, et al. Antioxidative stress-associated genes in circulating progenitor cells:evidence for enhanced resistance against oxidative stress. Blood 2004;104(12): 3591-3597 doi:10.1182/blood-2003-12-4103

27. Chen $L$, Tredget EE, Wu PY, et al. Paracrine factors of mesenchymal stem cells recruit macrophages and endothelial lineage cells and enhance wound healing. PLoS ONE 2008;3(4): e1886 doi:10.1371/journal.pone.0001886.

28. Tögel F, Hu Z, Weiss K ,et al. Administered mesenchymal stem cells protect against ischemic acute renal failure through differentiation-independent mechanisms. Am J Physiol Renal Physiol 2005;289(1):F31-42 doi:10.1152/ajprenal.00007.2005

\section{Figures}

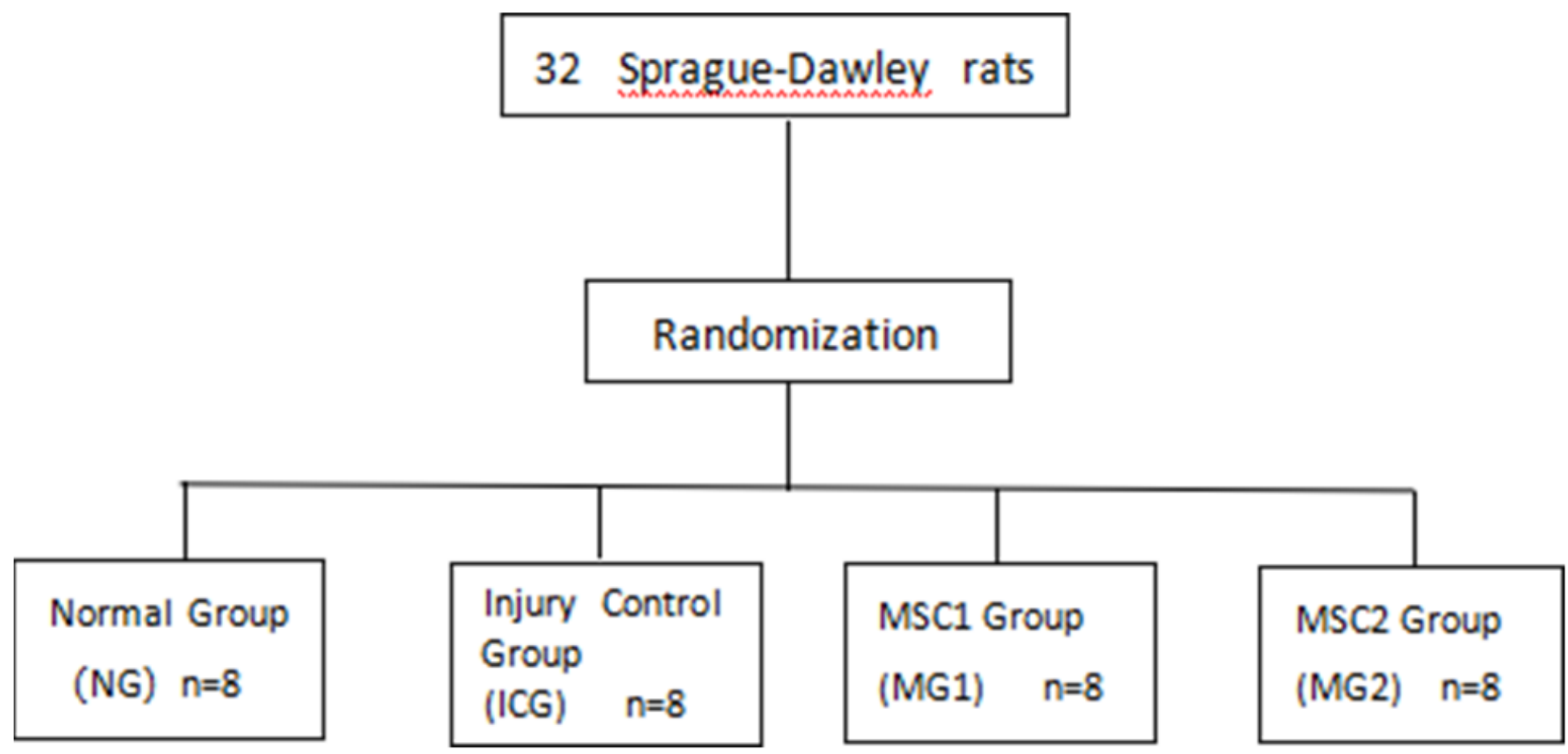

Figure 1

Transplantation of hUMSCs 


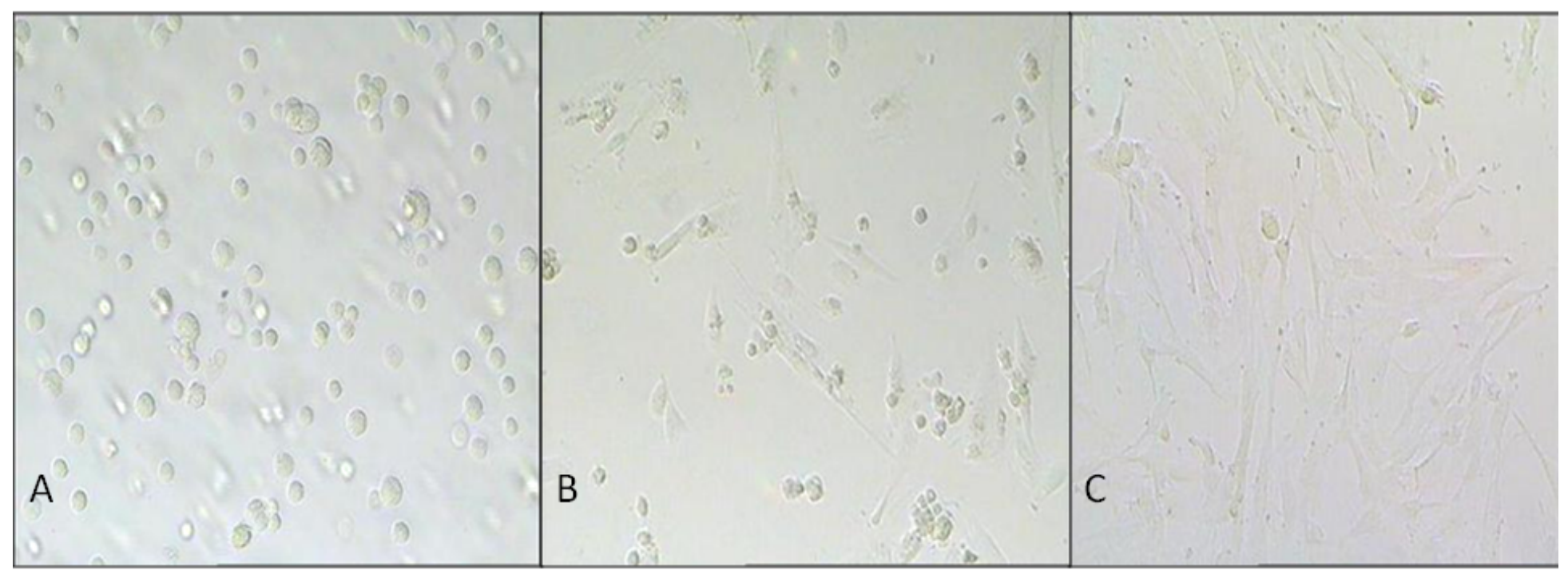

Figure 2

The morphology of hUCMSCs
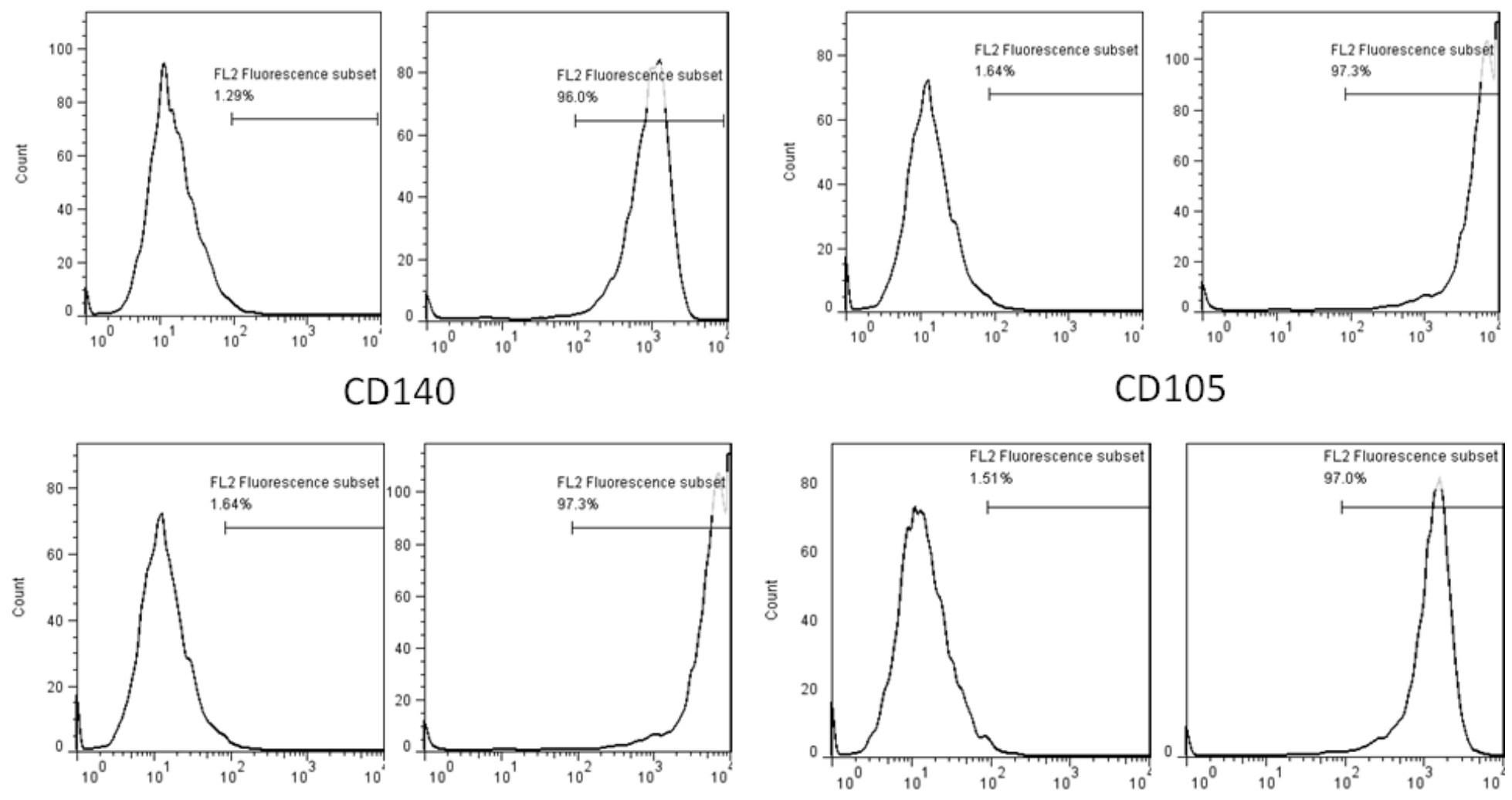

CD105

CD29

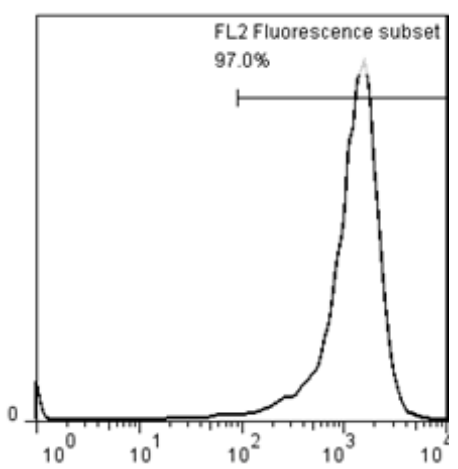

CD44

Figure 3

Expression levels of CD140, CD105, CD29 and CD44 in hUCMSCs 


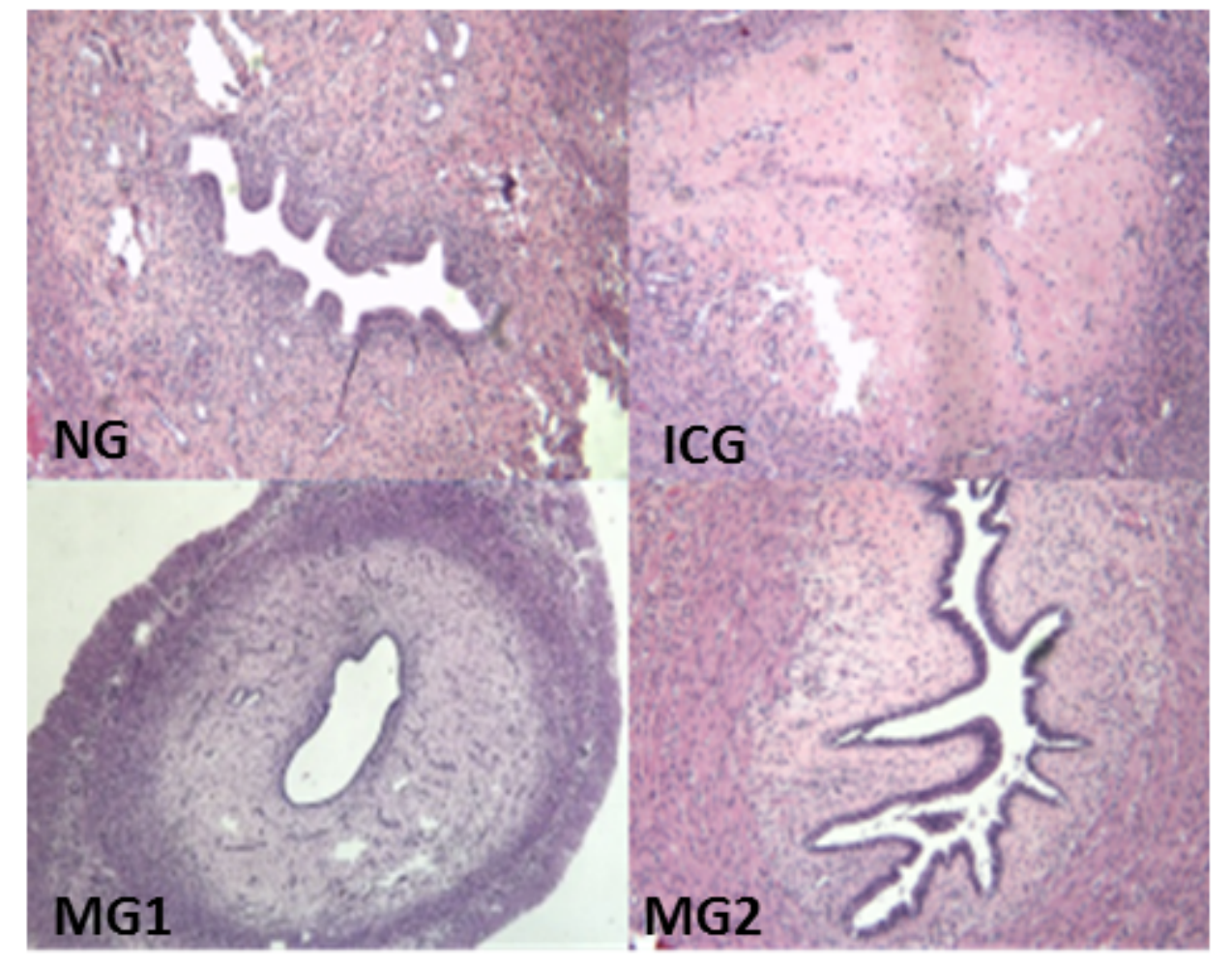

\section{Figure 4}

The results of rat endometrial Hematoxylin-Eosin Staining NG: The endometrial epithelium was cubic columnar in morphology and the intima was wrinkled. ICG: Endometrial injury, closed uterine cavity, no endometrial epithelium, glands and endometrial stroma. MG1: epithelial cells were seen in the uterine cavity, wrinkles decreased, endometrial cells and individual glands observed, regeneration of endometrial damage observed. MG2: Endometrial morphology and he normal group was similar and the endometrial damage was repaired significantly.

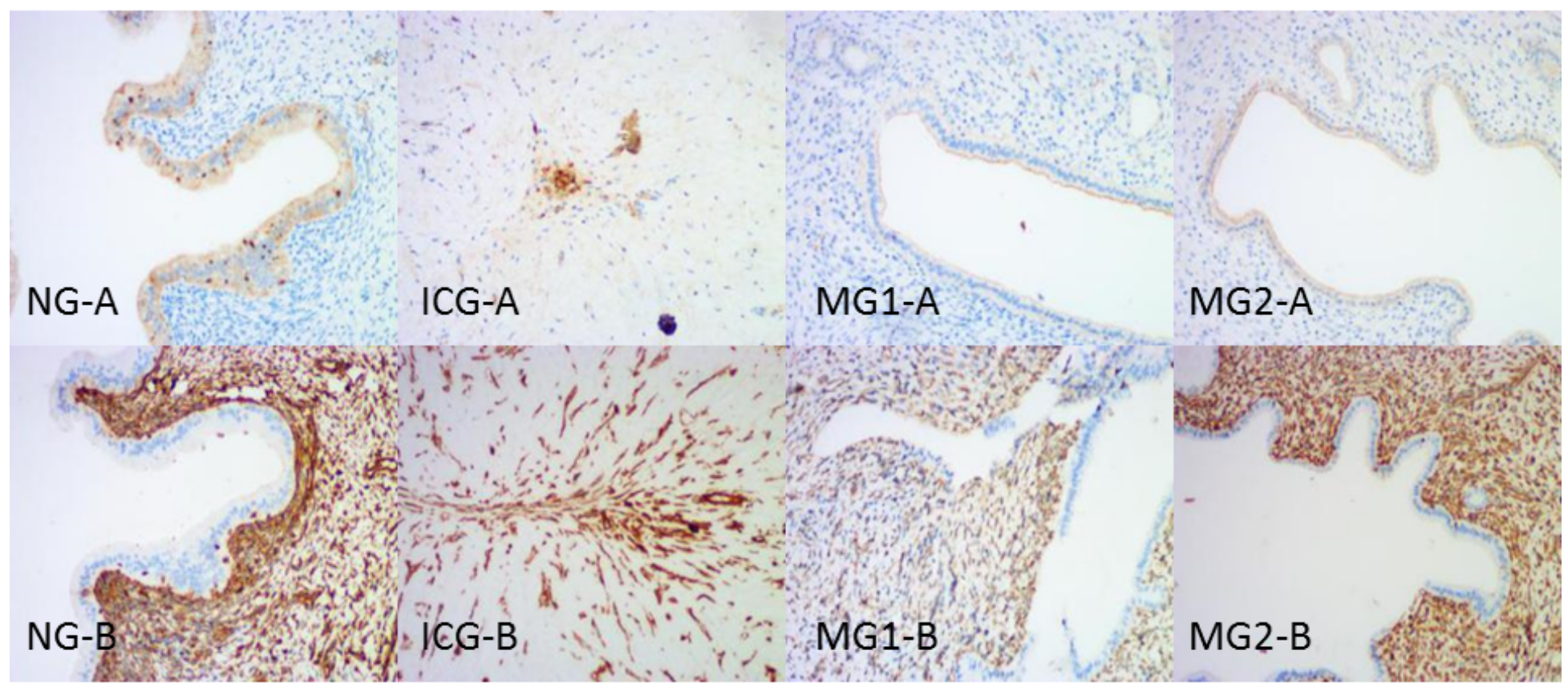


Figure 5

Panel A shows immunohistochemical staining of CK in each group, and panel B shows immunohistochemical staining of Vimentin in each group.
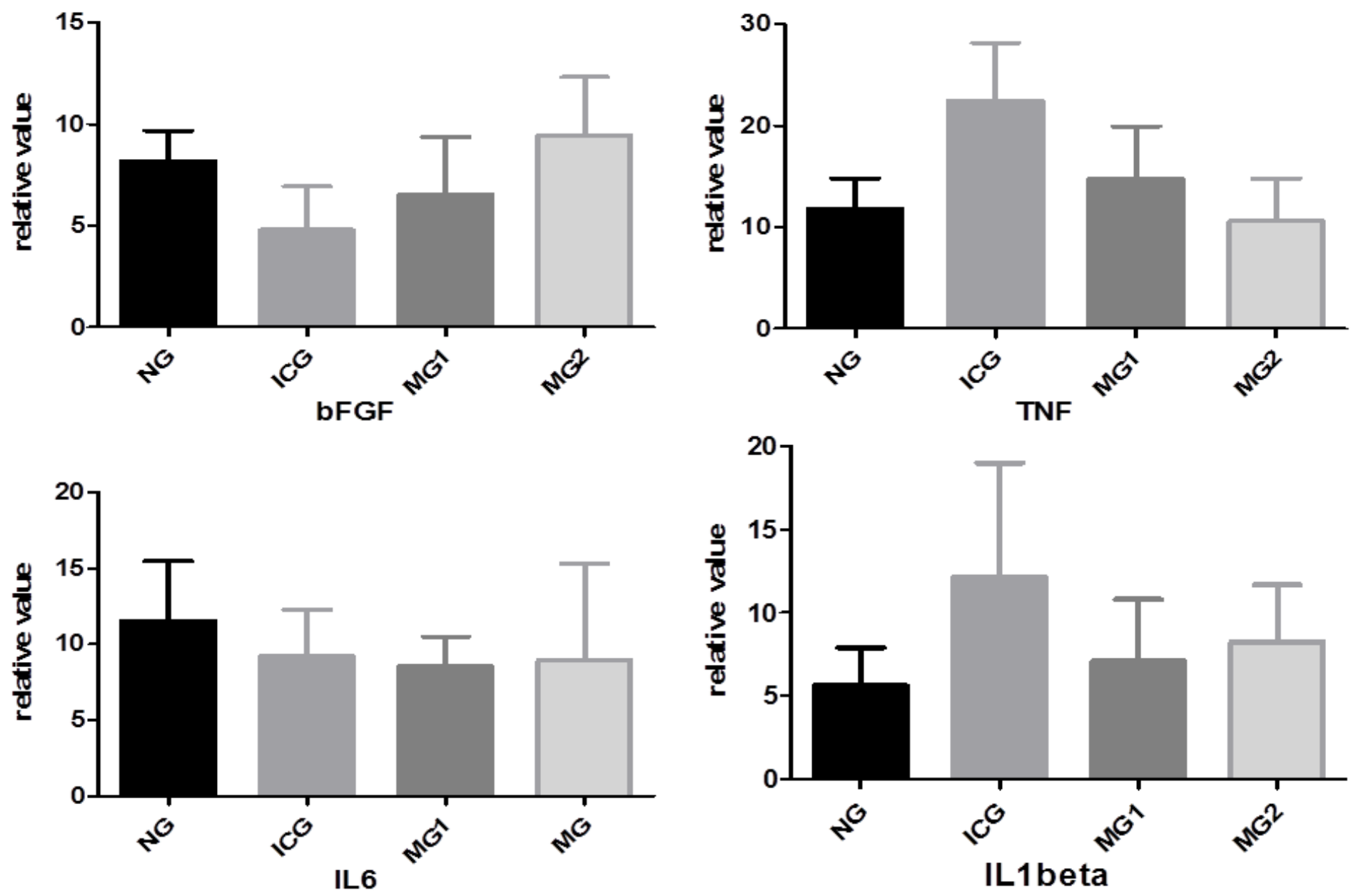

Figure 6

Figure 6: The expression levels of bFGF, TNF-a, IL-6 and IL-1 $\beta$ genes in the rat endometrium

\section{Supplementary Files}

This is a list of supplementary files associated with this preprint. Click to download.

- NC3RsARRIVEGuidelinesChecklistfillable.pdf 